\title{
A potential flow based flight simulator for an underwater glider
}

\author{
Surasak Phoemsapthawee ${ }^{a, *}$, Marc Le Boulluec ${ }^{b}$, Jean-Marc Laurens ${ }^{c}$, François Deniset ${ }^{d}$
}

\author{
a International Maritime College, Kasetsart University-Si Racha Campus, Si Racha, 20230, Thailand \\ ${ }^{\mathrm{b}}$ Ifremer, RDT/HO, BP-70, Plouzané, 29280, France \\ ${ }^{c}$ Ensta-Bretagne, 2 rue François Verny, Brest cedex9, 29806, France \\ d IRENav, Ecole Navale, Lanveoc, CC-600, Brest cedex9, 29240, France \\ *: Corresponding author : Surasak Phoemsapthawee, email address : $\underline{\text { surasak.phoemsapthawee@gmail.com }}$
}

\begin{abstract}
:
Underwater gliders are recent innovative types of autonomous underwater vehicles (AUVs) used in ocean exploration and observation. They adjust their buoyancy to dive and to return to the ocean surface. During the change of altitude, they use the hydrodynamic forces developed by their wings to move forward. Their flights are controlled by changing the position of their centers of gravity and their buoyancy to adjust their trim and heel angles. For better flight control, the understanding of the hydrodynamic behavior and the flight mechanics of the underwater glider is necessary. A 6-DOF motion simulator is coupled with an unsteady potential flow model for this purpose. In some specific cases, the numerical study demonstrates that an inappropriate stabilizer dimension can cause counter-steering behavior. The simulator can be used to improve the automatic flight control. It can also be used for the hydrodynamic design optimization of the devices.
\end{abstract}

Keywords: underwater glider ; potential flow ; Newton-Euler equation ; autonomous underwater vehicles (AUVs) ; flight simulator 


\section{Nomenclature}

\begin{tabular}{|c|c|}
\hline$B$ & Center of buoyancy $^{1}$ \\
\hline $\mathbf{E}_{3}$ & $3 \times 3$ Identity matrix \\
\hline$G$ & Center of gravity \\
\hline $\mathbf{I}_{m / O_{b}}$ & Matrix of moment of inertia \\
\hline$L_{w}$ & Main wing lift force \\
\hline$L_{s}$ & Stabilizer lift force \\
\hline M & $6 \times 6$ Matrix of inertia \\
\hline $\mathbf{M}_{a}$ & $6 \times 6$ Matrix of estimated added inertia \\
\hline$m$ & Glider mass \\
\hline$O_{b}$ & Origin of the body reference frame \\
\hline$O_{g}$ & Origin of the Galilean reference frame \\
\hline$R_{b}$ & Body reference frame \\
\hline$R_{g}$ & Galilean reference frame \\
\hline$\vec{V}_{G / R_{b}}$ & $\begin{array}{l}\text { Velocity of center of gravity with respect } \\
\text { origin of body reference frame }\end{array}$ \\
\hline$\vec{V}_{O_{b} / R_{b}}$ & Glider velocity in body reference frame \\
\hline$|V|$ & Total velocity $=\left(V_{X}^{2}+V_{Y}^{2}+V_{Z}^{2}\right)^{1 / 2}$ \\
\hline$x_{b}, y_{b}, z_{b}$ & Body reference frame coordinates \\
\hline$\nabla$ & Glider volume \\
\hline$\vec{\Omega}_{R_{b} / R_{g}}$ & Glider angular velocity \\
\hline$\rho$ & Water density $\left(1025 \mathrm{~kg} / \mathrm{m}^{3}\right)$ \\
\hline$\phi$ & Roll, Euler angle around $\mathrm{x}$-axis \\
\hline$\phi_{b}$ & Heel command angle \\
\hline$\theta$ & Pitch, Euler angle around y-axis \\
\hline$\theta_{b}$ & Trim command angle \\
\hline$\psi$ & Yaw, Euler angle around z-axis \\
\hline y & Torsor of force and moment \\
\hline $\mathscr{H}$ & Torsor of glider velocity in body reference frame \\
\hline \multicolumn{2}{|c|}{$\begin{array}{ll}\text { superscript } d o t & \text { Time derivative } \\
\text { subscript ext } & \text { Reference to external } \\
\text { subscript fic } & \text { Reference to fictitious }\end{array}$} \\
\hline
\end{tabular}

\section{Introduction}

Underwater gliders are a recent innovation of Autonomous Underwater Vehicles (AUVs) used in ocean exploration and observation. This type of AUVs allows for long-range and/or extended duration deployments; see its missions for examples in Bachmayer et al. (2004), Frajka-Williams et al. (2009) and Ramp et al. (2009). Nowadays there are three well-known underwater gliders on the market: Seaglider, Slocum and

Received date:

Foundation item:

*Corresponding author Email: jean-marc.laurens@ensta-bretagne.fr

(C) Harbin Engineering University and Springer-Verlag Berlin Heidelberg 2010
Spray (Eriksen et al., 2001 and Rudnick et al., 2004). However, they are still under development worldwide, for example Alvarez et al. (2009). In France, there are also developments of this AUVs type. Sterne is the underwater glider developed by Ensta-Bretagne (formerly Ensieta), Moitie and Seube (2001) and Phoemsapthawee et al. (2011).

The underwater gliders use small changes in their buoyancy to dive and to return to the ocean surface. During the change of altitude, the underwater gliders, like air gliders, use the hydrodynamic forces generated by their wings to move forward to the desired location. Since they use the buoyancy and the gravity force to propel themselves, their propulsion system consumes very low energy compared to the other AUVs. The low energy consumption of the propulsion system enables long duration operations. Studies are also carried out to use ocean thermal energy to propel underwater gliders in order to extend the operation time, for example Kong et al. (2010). Without any external moving part, except for the Slocum Electric which is equipped with an adjustable rudder, the underwater glider flights are controlled by changing the position of the center of gravity and/or buoyancy to adjust the trim and the heel angles, Bender et al. (2008).

Many research studies with respect to the automatic flight control of underwater gliders have been undertaken, for examples Bhatta and Leonard $(2002,2008)$. However, most (if not all) of the underwater glider automatic flight controls employ a quasi-static empirical hydrodynamic model, Graver et al. (2003) and Kan et al. (2008), and need hydrodynamic coefficients identifications, Graver (2005) and Geisbert (2007).

Sterne, the glider named after the seabird Sternula superciliaris, developed at Ensta-Bretagne, showed unexpected behaviour when equipped with a too small vertical stabilizer at its stern. As the internal mobile mass was displaced on starboard in order to force the glider to make it turn into the starboard direction, it did heel as expected but turned to port. The standard parametric model initially used did not anticipate such behaviour. Because the vertical stabilizer generates the moment allowing the glider to align with the circular trajectory, the simple practical solution was to increase its size. However, a team of hydrodynamicists formed by the authors of the present paper then attempted to find an explanation in order to correct the pilot and to avoid such unexpected behaviour which may cause the loss of the device.

Firstly, a new parametric model was developed with a solid and fluid inertia matrix (mass and added mass) which was properly computed according to the geometry and the mass distribution. The Euler-Newton equations solver was then coupled with a true unsteady hydrodynamic solver. The solver is an unsteady potential flow solver usually used for foils and 
propellers which is adapted for glider simulations. The potential flow model has a strong hypothesis but although it is, in principle, possible to couple the motion solver with a RANSE solver. Such a simulator consumes a lot of computation time and resource, see for example Murman et al. (2003), and is not suitable for simulations of longer duration. Moreover, the glider always operates at small angles of incidence and is not subject to significant flow separation. The assumptions of potential flow theory are thus respected and the friction forces are computed from the potential flow velocities. The computing time taken by this solver is of course important compared to the negligible time involved in the parametric model but remains reasonable.

Most simulations with both approaches produce comparable results. However, the counter steering behaviour of the glider equipped with a too small vertical stabilizer can only be obtained when the real hydrodynamic solver is used.

\section{Numerical model}

Since it is the principal aim of the numerical model to simulate the trajectory of the Sterne glider, the models and numerical tools have been especially developed for that purpose. In particular, the glider is a fully rigid body without any external mobile part. Like most gliders it is piloted using a ballast tank and a mobile inner mass to control its heel and trim angles.

\subsection{Dynamic model}

The equations of motion, also known as, the Euler-Newton equations are developed for a glider whose center of gravity, mass and moment of inertia can vary in the body reference frame. Since the variation of the glider inertia and center of gravity with respect to the body reference frame can be determined from the flight control command, the unknowns of the system are the translation and the angular accelerations $\left(\dot{\vec{V}}_{O_{b} / R_{b}}\right.$ and $\dot{\vec{\Omega}}_{R_{b} / R_{g}}$ ) of the body reference frame. The Euler-Newton equations can be written in matrix form as

$$
\left[\begin{array}{cc}
m \mathbf{E}_{3} & -m\left[O_{b} G\right] \\
m\left[O_{b} G\right] & \mathbf{I}_{m / O_{b}}
\end{array}\right]\left\{\begin{array}{c}
\dot{\vec{V}}_{O_{b} / R_{b}} \\
\dot{\vec{\Omega}}_{R_{b} / R_{g}}
\end{array}\right\}=\left\{\begin{array}{c}
\Sigma \vec{F}_{e x t} \\
\Sigma \vec{M}_{e x t}
\end{array}\right\}-\left\{\begin{array}{c}
\vec{F}_{f i c} \\
\vec{M}_{f i c}
\end{array}\right\}
$$

where $\left[O_{b} G\right]=\left[\begin{array}{ccc}0 & -z & y \\ z & 0 & -x \\ -y & x & 0\end{array}\right]$ is the skew-symmetric cross product matrix of $\overrightarrow{O_{b} G}=(x, y, z)^{T}$.

$\vec{F}_{f i c}$ and $\vec{M}_{f i c}$ are the fictitious force and moment and defined as

$$
\begin{aligned}
\vec{F}_{f i c}= & m \vec{\Omega}_{R_{b} / R_{g}} \times \vec{V}_{O_{b} / R_{b}}+m \vec{\Omega}_{R_{b} / R_{g}} \times\left(\vec{\Omega}_{R_{b} / R_{g}} \times \overrightarrow{O_{b} G}\right) \\
& +m \dot{\vec{V}}_{G / R_{b}}+2 m \vec{\Omega}_{R_{b} / R_{g}} \times \vec{V}_{G / R_{b}} \\
& +\dot{m} \vec{V}_{O_{b} / R_{b}}+\dot{m} \vec{\Omega}_{R_{b} / R_{g}} \times \overrightarrow{O_{b} G}+\dot{m} \vec{V}_{G / R_{b}} \\
\vec{M}_{f i c}= & \vec{\Omega}_{R_{b} / R_{g}} \times\left(\mathbf{I}_{m / O_{b}} \vec{\Omega}_{R_{b} / R_{g}}\right)+m \overrightarrow{O_{b} G} \times\left(\vec{\Omega}_{R_{b} / R_{g}} \times \vec{V}_{O_{b} / R_{b}}\right) \\
& +\dot{\mathbf{I}}_{m / O_{b}} \vec{\Omega}_{R_{b} / R_{g}}+\dot{m} \overrightarrow{O_{b} G} \times \vec{V}_{O_{b} / R_{b}}
\end{aligned}
$$

The external forces $\left(\vec{F}_{e x t}, \vec{M}_{e x t}\right)$ of the glider are the gravity forces, the buoyancy forces and the hydrodynamic forces $\left(\vec{F}_{\text {hydro }}, \vec{M}_{\text {hydro }}\right)$.

$$
\begin{gathered}
\vec{F}_{\text {ext }}=m \vec{g}-\rho \nabla \vec{g}+\vec{F}_{\text {hydro }} \\
\vec{M}_{\text {ext }}=m \overrightarrow{O_{b} G} \times \vec{g}-\rho \nabla \overrightarrow{O_{b} G} \times \vec{g}+\vec{M}_{\text {hydro }}
\end{gathered}
$$

Because of the mobile inner mass and the ballast tank, we allow the mass to vary with time and the inertia matrix $\mathbf{I}_{m / O_{b}}$ has to be recomputed at each time step. Since the ballast water has an impact on the mass but not on the outer body geometry, the added mass matrix and centre of buoyancy do not change.

\subsection{Hydrodynamic model}

An unsteady flow Boundary Element Method (BEM) code is used to compute the hydrodynamic forces $\left(\vec{F}_{h y d r o}, \vec{M}_{h y d r o}\right)$. In such methods, the flow is assumed potential, i.e. inviscid and irrotational. The boundary condition on the surface is a slip condition $(\vec{V} \cdot \vec{n}=0)$. To model lifting bodies such as the wings which present a sharp trailing edge, the Kutta-Joukowski boundary condition is applied. The spatial and time discretization sensitivity of the code are well mastered. The code has been verified and experimentally validated several times in the past, for example Phoemsapthawee et al. (2009). The code belongs to what Hoeijmakers (1992) refers to as "Second generation" panel methods involving a Dirichlet condition. The surfaces of both lifting and non-lifting bodies are discretized into first order panels carrying constant source $\sigma$ and doublet $\mu$ distributions. The wakes developed behind the lifting bodies are formed with sheets of first order panels carrying constant doublet distribution. The wings wake geometry is naturally described since it is generated in a Lagrangian manner.

Concerning the viscous effects, only the friction force is taken into account. Neither separation flow nor boundary layer effect is taken into account. The local friction coefficient $C_{f}$ is defined as a function of the local Reynolds number $\operatorname{Re}_{s}=V\left(s-s_{0}\right) / v$ where $s$ is the curvilinear coordinate, $s_{0}$ the stagnation location and $v$ 
the kinematic viscosity. If the local Reynolds number is less than $5 \times 10^{5}$, the flow is supposed to be laminar and the friction coefficient is taken from the Blasius solution, $C_{f}=0.664 / \sqrt{R e_{s}}$. Else, the flow is supposed to be turbulent and the friction coefficient is determined from an empirical formula, $C_{f}=0.027 / \sqrt[7]{R e_{s}}$. By integration over the panels, the resultant friction forces and moments are added to the hydrodynamic forces. Unlike the wings, the body does not present a sharp trailing edge, therefore it is assumed to be non-lifting. No wake develops behind it and the integrated pressure forces and moments are nil. To account for the flow inertia forces and moments, the added mass matrix is computed previously using a dedicated potential flow code. The added mass forces and moments are then computed from this matrix and the acceleration vector. Finally, the 1st-order Euler explicit numerical scheme is applied for the time variation in the potential flow computation.

As mentioned in the introduction, most of the underwater glider flight controls use a quasi-static empirical hydrodynamic approach for which the hydrodynamic coefficients were identified from experimental results. A quasi-static hydrodynamic model approach has also been written. In the simple hydrodynamic approach, the lift $L$ and the drag $D$ forces of the lifting bodies (main wing and stabilizer) are calculated from

$$
L=\frac{1}{2} \rho V^{2} A C_{L} \quad \text { and } \quad D=\frac{1}{2} \rho V^{2} A C_{D}
$$

where $V$ is the foil incident velocity and $A$ is the foil planform area. The lift coefficient $C_{L}$ and the induced drag coefficient $C_{D}$ are estimated using the Prandtl approximation:

$$
C_{L}=2 \pi \alpha \frac{\Lambda}{\Lambda+2} \quad \text { and } \quad C_{D}=\frac{C_{L}^{2}}{\pi \Lambda}
$$

where $\alpha$ is the angle of attack in radian and $\Lambda$ is the aspect ratio. When the glider rolls around its axis, the angles of attack seen by the sections along its wing span do not have a uniform distribution. The induced hydrodynamic moment can be estimated from the integration of $2 \mathrm{D}$ section lift as

$$
M=-\frac{\pi}{6} \rho V \omega c b^{2}
$$

where $\omega$ is the glider angular velocity along the glider axis, $c$ is the foil chord and $b$ is the foil span.

In addition to the pressure forces, friction forces must be taken into account. The principal drag force of the glider is the friction on the glider surface along the glider axis. The friction force is computed using the same formulae as previously but applied to an equivalent flat plane since the body geometry is not explicitly represented by a surface mesh. The added mass forces and moments of the main body are computed as for the BEM code and are ignored for the lifting bodies (wings and stabilizer).

In summary: the simple parametric hydrodynamic model includes lift and drag generated by the lifting bodies (main wing and stabilizer) for a steady state flow. The main wing spanwise hydrodynamic moment generated by the glider heeling motion is also included. The friction forces on the lifting bodies are ignored. The axial friction force on the main body is computed as a function of the Reynolds number and is applied to the centre of buoyancy. The added mass matrix is computed with a dedicated potential flow code and the added mass forces only depend upon the acceleration vector. Gravity and buoyancy forces and moments are included in the dynamic model.

The potential model computes lift, drag and moment generated by the lifting bodies (main wing and stabilizer) including the unsteady component and the friction. The friction force and moment on the main body are computed by the integration of the friction force over the panels computed as a function of the Reynolds number. As for the parametric model, the added mass matrix is computed with the dedicated potential flow code and the added mass forces only depend upon the acceleration vector. Gravity and buoyancy forces and moments are included in the dynamic model.

The main difference between the two hydrodynamic codes lies in the way the lifting bodies (wings and stabilizer) are modelled: fully unsteady in the potential flow code and quasi-static in the parametric code. The main body is explicitly modelled in the BEM code. Hence its effect on the wing is taken into account and the friction forces and moments can be computed while the friction effect is not much more than a drag coefficient in the parametric model.

\subsection{Dynamic-hydrodynamic coupling method}

The simulation scheme is explained here. The parametric simulator can easily start with a zero initial velocity. At first, only the gravity force causes the motion but the hydrodynamic forces build up once the glider starts to move. Starting the potential solver with a zero initial velocity is also possible but unnecessarily time consuming. The best method is to initially set the glider with a small non-zero velocity to mimic the operator pushing it to launch it. The hydrodynamic model is solved first in order to obtain the hydrodynamic forces in the initial conditions. Starting from the initial conditions (positions, velocities, hydrodynamic forces), the Euler-Newton equations are solved to obtain the new positions and the new velocities using the 4th-order Runge-Kutta method. After the Euler-Newton solver, the hydrodynamic forces are calculated from the new positions 
and the new velocities using the potential flow solver. The two solvers are independent from each other. Once the hydrodynamic forces are updated, the next time step is considered and the Euler-Newton equations are recalculated. The process continues in this way until the end of simulation. Fig. 1 presents the flow chart of this simulation procedure. The size of the time step is dictated by the potential flow code requirement.

The error of the added inertia calculation in the hydrodynamic forces can cause the instability of the motion calculation (Floc'h et al. 2008). This numerical error can be reduced by adding the added inertia on both sides of the Euler-Newton equations as shown in a torsor form as

$$
\left.\left(\mathbf{M}+\mathbf{M}_{a}\right) \dot{\mathcal{U}}\right|_{n}=\left.\mathscr{\mathcal { F }}_{\text {ext }}\right|_{n}-\left.\mathscr{F}_{f i c}\right|_{n}+\left.\mathbf{M}_{a} \dot{\mathcal{U}}\right|_{n-1}
$$

where $\mathbf{M}_{a}$ is the estimated added-inertia matrix with respect to the body reference frame and $n$ is the time step number.

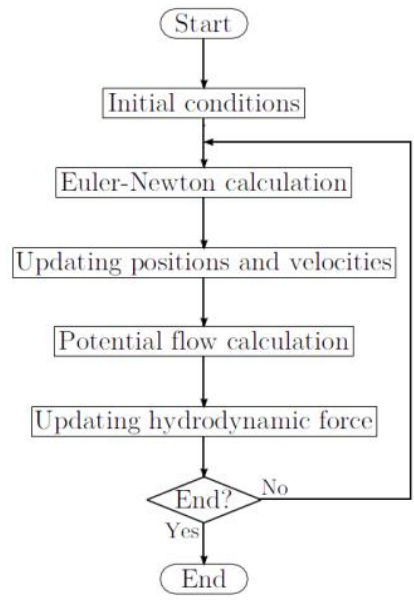

Fig.1 Simulation scheme chart

The stability of this numerical error reduction scheme is discussed in Floc'h et al. (2008). The article demonstrates that a large range of estimated added inertia produces the same motion as the genuine added inertia. In this paper, the estimated added inertia is pre-calculated by a non-lifting potential flow code using Rankine singularities.

\section{Numerical simulation}

The glider geometry is presented in Fig.2. The glider body can be separated into three parts: the head, the main body and the tail. The main body is a cylinder with a $0.2 \mathrm{~m}$ diameter and $1 \mathrm{~m}$ length. The head and the tail are hemi-ellipsoids with the same diameter as the main body and have a length of $0.5 \mathrm{~m}$. The wing and the stabilizer section profiles are NACA0005. The volume and the mass of the lifting parts are neglected in the simulations. Hence, the glider volume and the displacement mass are solely calculated based on the glider body and the center of buoyancy $B$ is positioned at the middle of the glider body. The ballast can take in or drain off the water of $\pm 0.5 \%$ of the glider volume. The glider moment of inertia is defined to be equal to the moment of inertia of a $0.2 \mathrm{~m}$ diameter and $2.0 \mathrm{~m}$ length cylinder of which the mass is equal to the glider displacement.

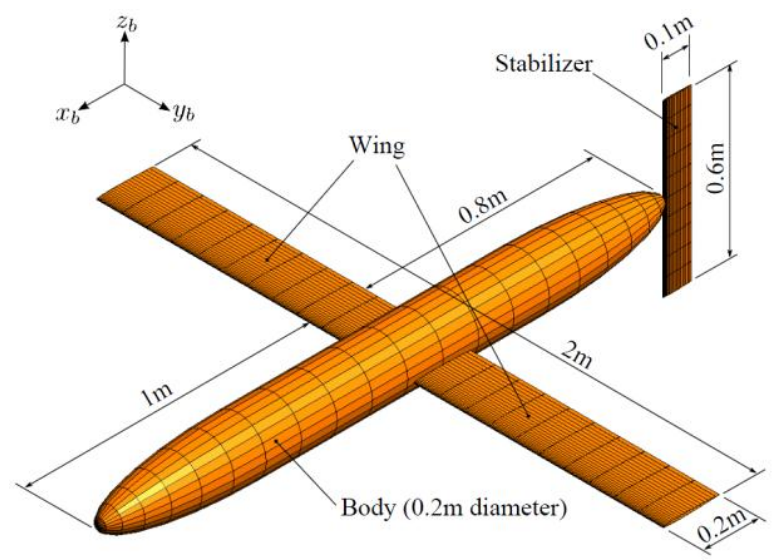

Fig.2 Glider model geometry

The position of the center of gravity of the glider is the resultant of the fixed mass, the mass of ballast water and a moving mass. In the model, the resultant center of gravity $G$ is directly considered. The moving mass can rotate around the glider axis at the fixed radius. It can also slide along the glider axis. As a result, the distance between the resultant center of gravity and the glider axis is constant. The glider flight control command consists therefore of two components: the buoyancy control and the position of the center of gravity control. The position of the center of gravity can be represented by two angles: the heel command angle $\phi_{b}$ and the trim command angle $\theta_{b}$. The definitions of the two angles are illustrated in Fig.3. In all simulations, the glider is initially launched with a $0.2 \mathrm{~m} / \mathrm{s}$ horizontal velocity in the $x_{b}$ - positive direction.

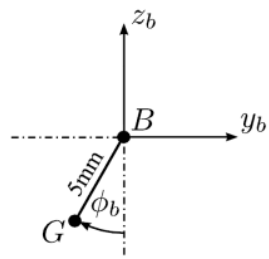

Front view

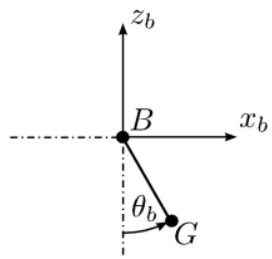

Starboard view
Fig.3 Definition of heel command angle $\phi_{b}$ and trim command angle $\theta_{b}$

\subsection{Sawtooth trajectory}

First, we simulate the glider advancing in sawtooth trajectories. The ballast and the trim command angle are varied alternatively. When the ballast takes in the water $(+0.5 \% \nabla)$, the trim command is positive; we call these conditions the command state I. When the ballast drains out the water $(-0.5 \% \nabla)$, the trim command is negative; we call these conditions the command state II. Each command state 
lasts 99 seconds. The glider conditions are varied between the command state I and II in a sinusoidal manner to avoid a sharp variation. The transition time lasts 1 second. The variation between state I and II is illustrated in Fig.4. This sawtooth trajectory is simulated for different trim command angles $\theta_{b}$ of $\pm 10, \pm 20, \pm 30$ and \pm 40 degrees.

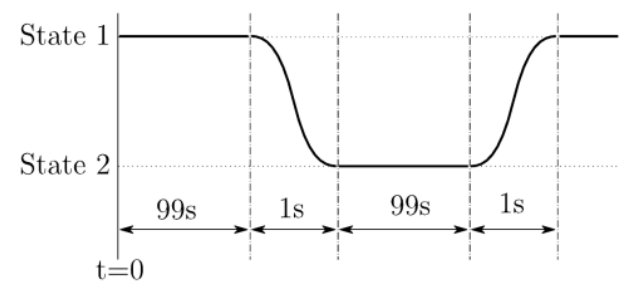

Fig.2 Glider model geometry

Both hydrodynamic approaches yield comparable results. Fig.5 shows the pitch response corresponding to each trim command angle. The pitch angle oscillates before reaching a steady state. In the case of the simple approach, the steady pitch angle is a little larger than the trim command angle due to the hydrodynamic moment generated by the main wing. However, in the case of the potential flow approach, the interaction between the main wing and the glider body produces a smaller steady pitch angle than the trim command angle.
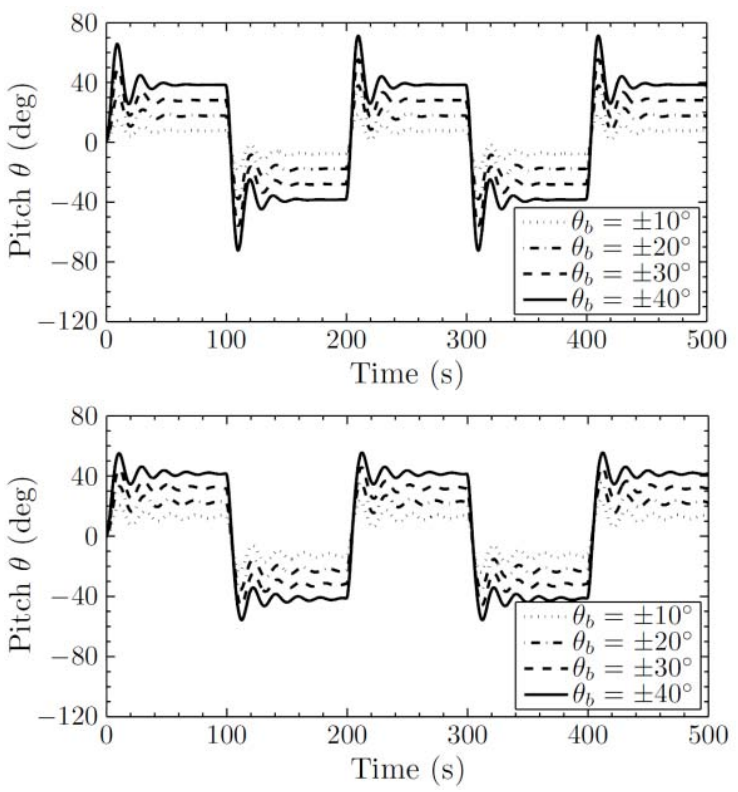

Fig.5 Pitch $\theta$ responses for different trim command angle $\theta_{b}$; top with potential flow approach, bottom with simple approach

The glider velocities are presented in Fig.6 and Fig.7. The simulation results confirm the relationship between the trim command angle and the steady velocity. As expected, the glider velocity increases with the trim command angle. The effect asymptotically converges for trim command angles between 30 and 40 degrees. Both hydrodynamic models agree that the horizontal velocity limit is reached when the trim command angle is around 40 degrees. However, the parametric model predicts a smaller horizontal velocity limit since it does not increase beyond a trim command angle of 30 degrees. The velocity differences between the two approaches depend without any doubt on the friction computation.

The angles of attack of the main wing presented in Fig.8 confirm the small incident flow assumption for both approaches. In this case, for the potential flow approach, the angle of attack is less than 6 degrees at the beginning of the simulations and less than 3 degrees at each command state variation. For the simple approach, the angles of attack are even smaller.

Fig.9 presents the sawtooth trajectories obtained by the two approaches for a 500 second simulation. Both approaches produce very similar results as expected. This will be the case for most trajectories simulations but in the next section, we will present a case where both approaches yield very different results.
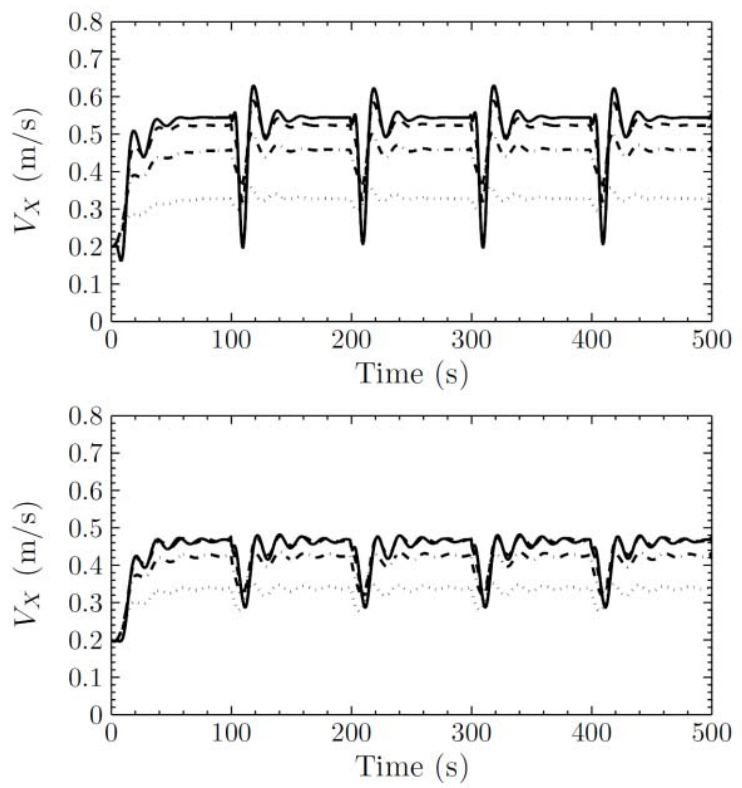

Fig.6 Horizontal velocity $V_{x}$ responses for different trim command angle $\theta_{b}$; top with potential flow approach, bottom with simple approach

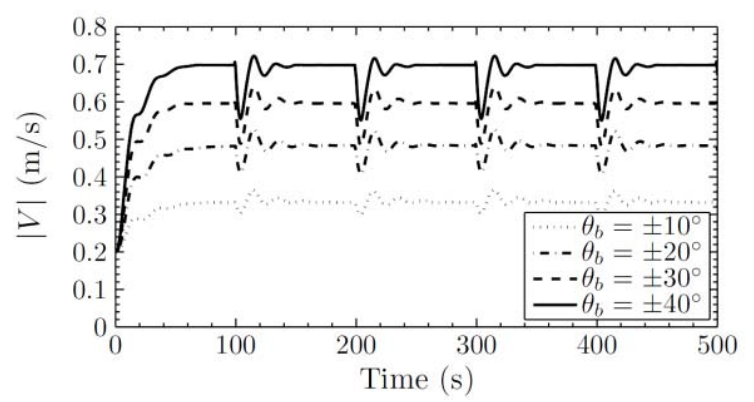




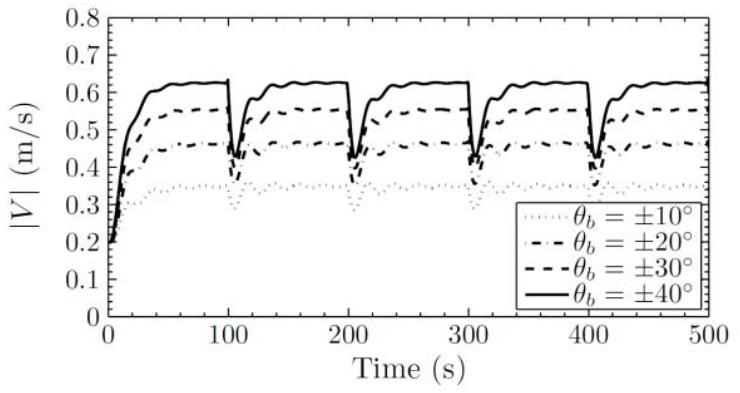

Fig.7 Total velocity $|V|$ responses for different trim command angle $\theta_{b}$; top with potential flow approach, bottom with simple approach

\subsection{Helical trajectory}

In the case presented in this section, we command the glider to dive with positive trim and heel command angles with the intention to change the glider direction. In the example presented here, trim and heel command angles are both equal to 10 degrees. Three different geometries of the stabilizer are considered. The dimensions of the three stabilizers are illustrated in Fig. 10.
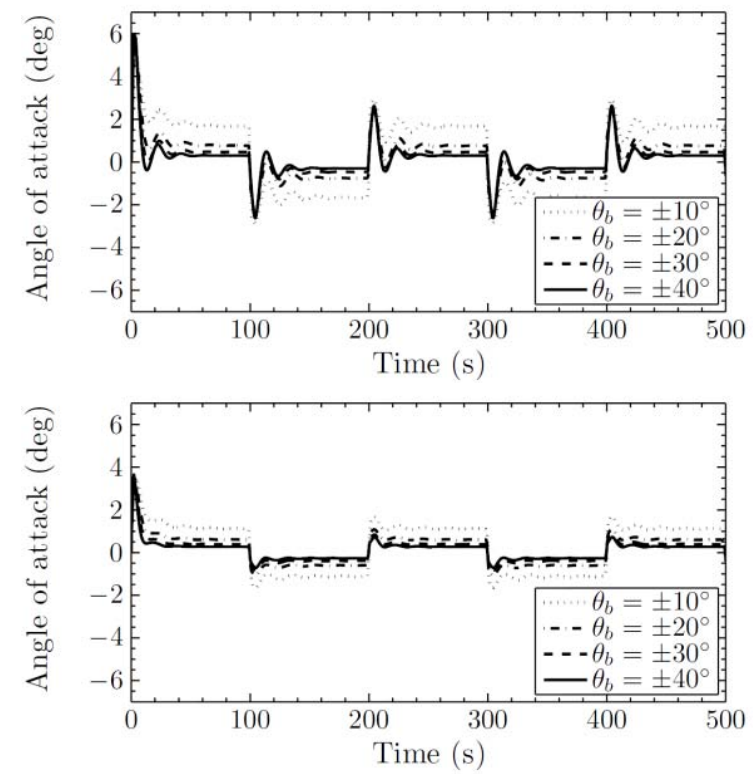

Fig.8 Angle of attack responses for different trim command angle $\theta_{b}$; top with potential flow approach, bottom with simple approach

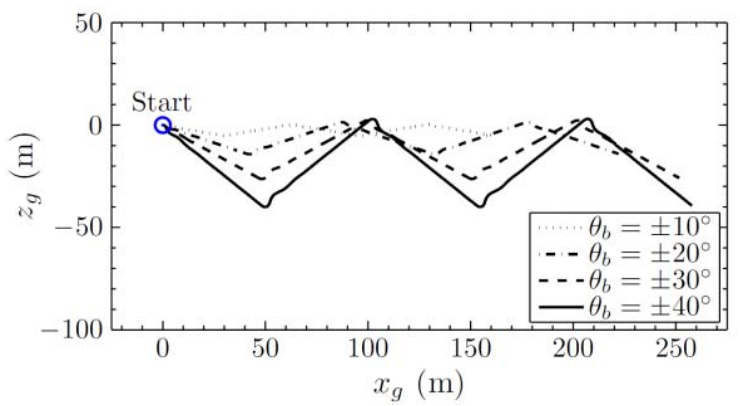

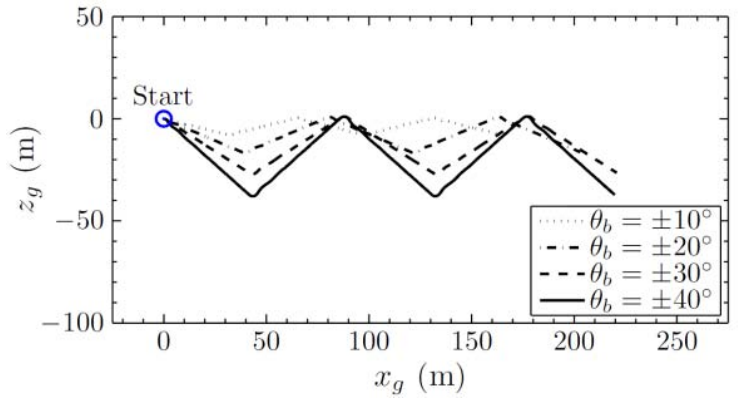

Fig.9 Sawtooth trajectory during 500 seconds for different trim command angle $\theta_{b}$; top with potential flow approach, bottom with simple approach

The glider is expected to turn because of the lateral component of the main wing lift force when the glider is heeling. In the case of a positive heel angle as in these simulations, the glider is then expected to turn to the starboard direction. The obtained trajectories for both approaches are presented in Fig.11. Unlike the sawtooth simulations, the two approaches produce very different results.
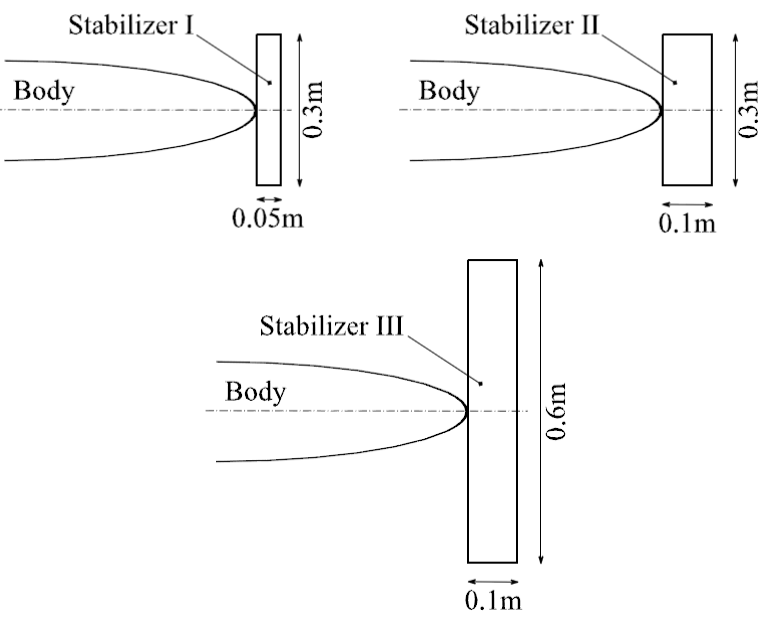

Fig.10 Dimensions of stabilizers

According to the numerical results of the simple approach, the glider moves to starboard as expected. The stabilizer size is only of little effect; the three trajectories are almost the same. The glider turns with the steering force generated by the main wing and the stabilizer generates the hydrodynamic moment to adjust the glider orientation to the incident inflow. However, this behaviour is not always observed in reality. The glider Sterne (Ahmed-Ali et al. 2003) which was developed at Ensta-Bretagne experienced counter-steering behaviour. The first Sterne model was lost during an experiment at sea because of this unexpected behaviour. It was suspected that the stabilizer size was responsible for this. The new Sterne model equipped with a larger stabilizer does not present any counter-steering behaviour. This is the reason why, in order to confirm the role of the stabilizer, we decided to cover different stabilizer geometries in this study. 
The potential flow results retrieve the experimentally observed counter-steering behaviour. The turning equilibrium conditions of the three stabilizers are compared in Table 1. These numerical results confirm that the stabilizer size plays an important role in this behaviour. The smallest stabilizer (stabilizer I) causes the glider to turn to the counter-steering direction (the port direction in this case) while the other two do not. When the stabilizer is large enough (stabilizer III), the glider behaves as expected and predicted by the simpler approach. In the case of the intermediate size (stabilizer II), the stabilizer is not large enough to steer the glider properly. When the stabilizer is too small, it cannot produce enough hydrodynamic moment to counteract the counter-steering hydrodynamic moment. The counter-steering hydrodynamic moment is generated by the main wing lift lateral component pointing to starboard and the lateral force due to the body drifting pointing to port. If not sufficiently countered, the moment will make the glider Sterne to turn to port because the centre of volume is situated well in front of the wing, see Fig.12.

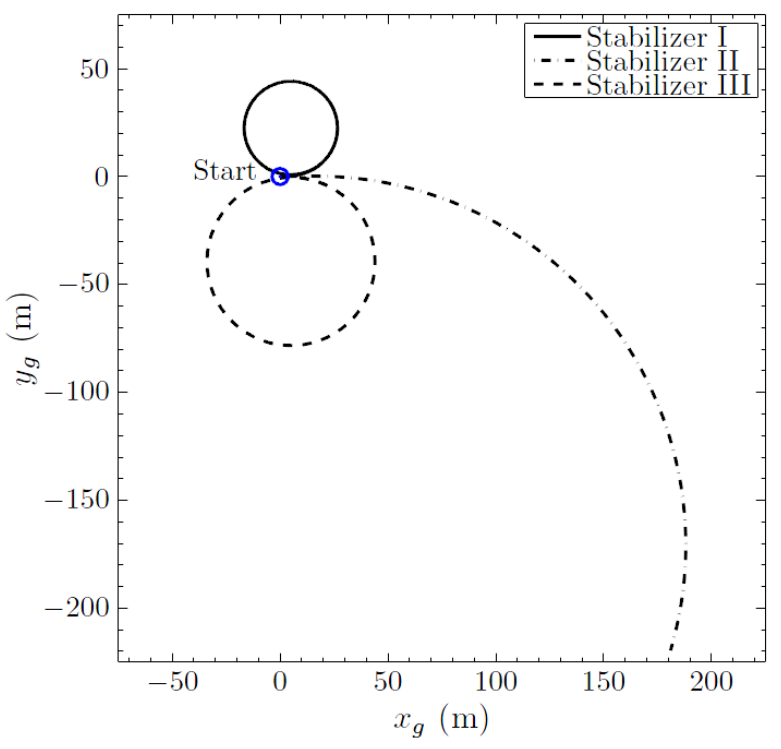

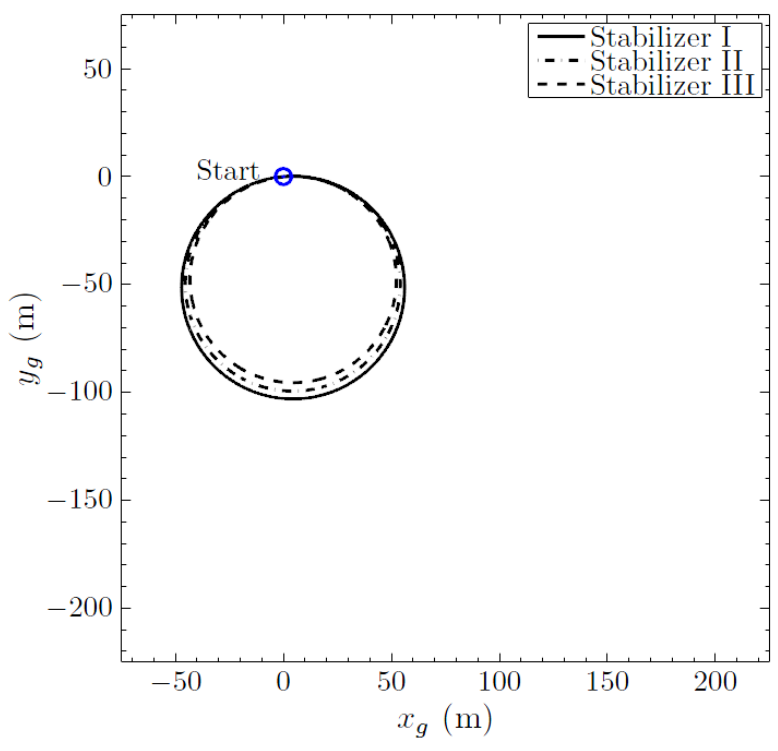

Fig. 11 Top view of helical trajectory during 1000 seconds for $10^{\circ}$ trim and $10^{\circ}$ heel command angles; top with potential flow approach, bottom with simple approach

In this case the source of the counter-steering moment was hence generated by the lateral friction force on the drifting body. The simple hydrodynamic model does not have this lateral force because as described in section 2.2, in this simple parametric model, the friction is only applied along the main axis. Once it starts to turn to port, the stabilizer sees a flow from the port direction and its lift points to starboard generating a moment at port helping the glider to find a stable route in the port direction.

With higher accelerations, the added mass tensor could take over this effect. The way the body added mass tensor is taken into account is the same for both models. In such cases, both models would see the same counter steering moment but it does not necessarily imply that they would predict the same trajectory because of the fluid inertia effect on the lifting bodies. In the simple model, the hydrodynamic moment generated by the stabilizer is fully perceived as soon as the glider heels without any delay. In the potential flow simulations, there is a delay between the geometric position and the hydrodynamic response. This delay exists because of the fluid inertia applied by the stabilizer. Because of this delay, if the stabilizer counteracting moment is not strong enough, the glider could find the time to position itself in the other equilibrium state causing the counter-steering behaviour.

Table 1 Turning equilibrium conditions from the potential flow approach results; the gliding diving with $10^{\circ}$ trim and $10^{\circ}$ heel command angle

\begin{tabular}{lccc}
\hline Stabilizer & I & II & III \\
\hline Turning radius $(\mathrm{m})$ & 22 & 171 & 39 \\
Turning rate $(\mathrm{deg} / \mathrm{s})$ & 0.88 & -0.11 & -0.48 \\
\hline
\end{tabular}




\begin{tabular}{lccc}
\hline Advance speed (m/s) & 0.334 & 0.339 & 0.329 \\
Diving speed (m/s) & 0.062 & 0.058 & 0.056 \\
AOA of stabilizer $(\mathrm{deg})$ & 8.85 & 3.95 & 2.35 \\
AOA of main wing $(\mathrm{deg})$ & 1.68 & 1.55 & 1.63 \\
Heel angle (deg) & 12.56 & 9.68 & 8.51 \\
Trim angle $(\mathrm{deg})$ & 7.08 & 7.51 & 7.67 \\
\hline
\end{tabular}

In principle, it is possible to add such effects and other effects in a parametric model. For instance, we could add the interaction between the glider body and its appendages using a correction factor as suggested by Caldeira and Clarke (1988). Adding some of these parameters would not significantly increase CPU time, but it would rapidly make the parametric model look like a patchwork difficult to control and to maintain. Before any additional parameter is included within the parametric model, its importance and effect have to be clearly identified. Furthermore, not all effects are easily replaced by a simple model involving a single parameter. The lateral friction drag for instance depends on the Reynolds number but also on the body geometry. Although the potential flow simulator does not take all the physics into account, it can be used as a numerical towing tank facility to improve the simulator, i.e. the parametric model. Its CPU time is not negligible compared to the simple hydrodynamic code but it consists in a stage between the parametric model and the experimental trials.

\section{Computation resource consumption}

We now consider the underwater glider equipped with the stabilizer III to simulate a non trivial trajectory. The objective is to simulate a helical diving trajectory followed by a surfacing contra-rotating helical trajectory. Like in the previous simulations, the glider is launched with a $0.2 \mathrm{~m} / \mathrm{s}$ horizontal velocity in the $x_{b}$-positive direction. The heel command angle is fixed at 20 degrees. The trim command and the ballast are varied alternatively every 1100 seconds. For the first 1100 seconds, the trim command angle is 10 degrees and the ballast takes the water in for diving. For the next 1100 seconds, the trim command angle is -10 degrees and the ballast drains the water off for surfacing. The total duration of the simulation is 2332 seconds and takes 20000 time steps. This simulation involves about 24 hours of computation time on a standard workstation (CPU 4 cores with $2.66 \mathrm{GHz}$ ).

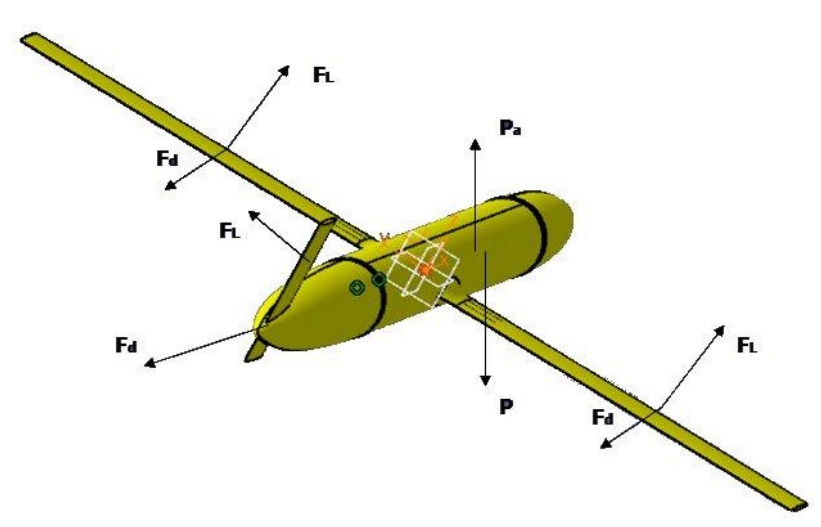

Fig.12 Steering mechanics: when inclined, the main wing lift presents a transversal component. The glider tends to drift toward the heel direction generating a drag force. Because this drag force center is in front of the wing, it produces a counter-steering moment. The role of the stabilizer is to counteract this moment.

\section{Conclusion and perspective}

An Euler-Newton equations solver is coupled with a potential flow code to simulate 6-DOF trajectories of underwater gliders. This simulator can be used to study the hydrodynamic behaviour of gliders in order to improve the automatic flight control and to optimize the geometry of the glider. A numerical study of the hydrodynamic behaviour of an underwater glider has been conducted. All results are compared with a simple parametric simulator. A series of simulations considering sawtooth trajectories has first been conducted. In this case both simulators present very similar trajectories. As expected, the glider velocity varies as a function of the trim angle. To confirm a behaviour observed experimentally, a second series of simulations concerning the glider steering has been launched. The potential flow simulator shows that the stabilizer geometry plays an important role in steering control. An inappropriate stabilizer geometry can cause counter-steering behaviour that the parametric simulator cannot anticipate. A non trivial case has finally been presented to demonstrate the capabilities of the potential flow based glider simulator. Enhancing the parametric model with additional features is easy but simple models are the best way to determine which physical effect is really important. The potential flow code is a good practical tool to simulate complex trajectory patterns and to verify whether the parametric code predicts the same trajectory. However, further validations using real data and experimental model testing are necessary to increase our confidence in the potential simulator before it can be adopted as a template for the parametric model enhancements. 


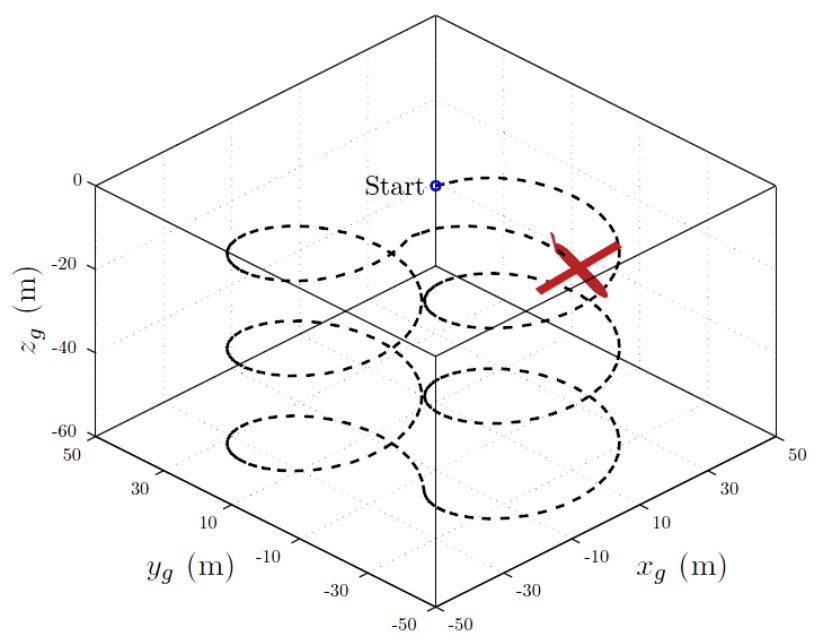

Fig.13 Example of trajectory simulation for a real-time duration of 2332 seconds. The CPU time in this case is about 24 hours on a standard PC. The glider appears 10 times bigger than it is.

Good quality experimental results will be extremely difficult to obtain. The behaviour of the Sterne glider has been obtained from sea trials. The onboard instruments record the velocities, depth, heel and pitch angles. The trajectory is deduced from these data. We have no information concerning the environmental conditions at sea. To conduct a proper experimental investigation at scale 1 , we would need a calm pool of water with very large dimensions. Although the glider is only 2 metres long, its gyration radius is about 30 metres. In Fig.13, the glider appears much larger than in reality as stated in the caption. We plan to perform some experimental trials on a reduced size model at the Ifremer tank facility in Brest. Since the scale cannot be too small, we will not be able to conduct anything resembling the case of Fig.13 in the tank. However, a full gyration is not necessary to study the counter-steering behaviour. The scale of the experimental model is the key issue since it will be a compromise between several factors including the Reynolds number, manufacture and the observable trajectories in the Ifremer towing tank facility.

\section{Acknowledgement}

This work is partially supported by the Europle Mer (http://www.europolemer.eu).

\section{References}

Ahmed-Ali T, Cuilerier L, Seube N (2003). Glider observer and identifier based on sliding modes control. Proceedings of Manoeuvring and Control, Girona, Spain.

Alvarez A, Caffaz A, Caiti A, Casalino G, Gualdesi L, Turetta A, Viviani R (2009). A low-cost autonomous underwater vehicle combining glider and AUV capabilities. Journal of Ocean Engineering, 36, 24-38.

Bachmayer R, Leonard NE, Graver J, Fiorelli E, Bhatta P, Paley D (2004). Underwater gliders: recent developments and future applications. Proceedings of the IEEE International Symposium on Underwater Technology (UT'04), Taipei, Taiwan.

Bender A, Steinberg DM, Friedman AL, Williams SB (2008). Analysis of an autonomous underwater glider. Proceedings of the Australian Conference on Robotics and Automation.

Bhatta P, Leonard NE (2002). Stabilization and coordination of underwater gliders. Proceedings of the $41^{\text {st }}$ IEEE Conference on Decision and Control.

Bhatta P, Leonard NE (2008). Nonlinear gliding stability and control for vehicles with hydrodynamic forcing. Automatica, $\mathbf{4 4}$ 1240-1250.

Caldeira-Savaira F, Clarke D (1988). The active control of swath motions. Proceedings of International Conference of Swath Ships and Advanced Multihulled Vessels II.

Eriksen CC, Osse TJ, Light RD, Wen T, Lehman TW, Sabin PL, Ballard JW, Chiodi AM (2001). Seaglider: a long-range autonomous underwater vehicle for oceanographic research. IEEE Journal of Oceanic Engineering, 26, 424-436.

Floc'h F, Laurens JM, Leroux JB, Kerampran S (2008). Trajectory prediction by coupling Euler-Newton equations and flow models. Proceedings of $11^{\text {th }}$ Numerical Towing Tank Symposium, Brest, France.

Frajka-William E, Rhines PB, Eriksen CC (2009). Physical controls and mesoscale variability in the Labrador sea spring phytoplankton bloom observed by Seaglider. Journal of Deep Sea Research Part I: Oceanographic Research Papers, 56, 2144-2161

Geisbert JS (2007). Hydrodynamic modeling for autonomous underwater vehicles using computational and semi-empirical methods. Master's thesis, Virginia Polytechnic Institute and State University.

Graver JG (2005). Underwater gliders: dynamics, control and design. Ph.D. thesis, Princeton University.

Graver JG, Bachmayer R, Leonard NE, Fratantoni DM (2003). Underwater glider model parameter identification. Proceedings of the $13^{\text {th }}$ International Symposium on Unmanned Untethered Submersible Technology (UUST).

Hoeijmakers H (1992). Panel methods for aerodynamic analysis and design. AGARD Report No.783, 5.1-5.47.

Kan L, Zhang Y, Fan H, Yang W, Chen Z (2008). MATLAB-based simulation of buoyancy-driven underwater glider motion. Journal of Ocean University of China, 7, 113-118.

Kong Q, Ma J, Xia D (2010). Numerical and experimental study of the phase change process for underwater glider propelled by ocean thermal energy. Journal of Renewable Energy, 35, 771-779.

Moitie R, Seube N (2001). Guidance and control of an autonomous underwater glider. Proceedings of the $12^{\text {th }}$ International Symposium on Unmanned Untethered Submersible Technology, Durham, NH, USA.

Murman SM, Aftosmis MJ, Berger MJ (2003). Simulations of 6-DOF motion with a cartesian method, Proceeding of $41^{s t}$ AIAA Aerospace Sciences Meeting, Reno, NV.

Phoemsapthawee S, Leroux JB, Laurens JM, Deniset F (2009). A transpiration velocities based sheet cavitation model. Ship Technology Research / Schiffstechnik, 56, 161-176.

Phoemsapthawee S, LeBoulluec M, Laurens JM, Deniset F (2011). Numerical study on hydrodynamic behavior of an underwater glider. Proceedings of the $30^{\text {th }}$ International Conference on Ocean, Offshore and Arctic Engineering OMAE-2011, Rotterdam, Netherlands.

Ramp S, Davis R, Leonard N, Shulman I, Chao Y, Robinson A, Marsden J, Lermusiaux P, Fratantoni D, Padaun J, Chavez F, 
Bahr F, Liang S, Leslie W, Li Z (2009). Preparing to predict: the second autonomous ocean sampling network (AOSN-II) experiment in the Monterey bay. Journal of Deep Sea Research Part II: Topical Studies in Oceanography, 56, 68-86.
Rudnick DL, Davis RE, Eriksen CC, Fratantoni DM, Perry MJ (2004). Underwater gliders for ocean research. Marine Technology Society Journal, 38, 48-59. 